\title{
Differential modulation of IL-12 family cytokines in autoimmune islet graft failure in mice
}

\author{
Feng-Cheng Chou ${ }^{1} \cdot$ Heng-Yi Chen ${ }^{2} \cdot$ Hsin-Hui Chen ${ }^{3}$ Gu-Jiun Lin $^{4} \cdot$ Shih-Hua Lin ${ }^{5}$. \\ Huey-Kang Sytwu ${ }^{1,2}$
}

Received: 6 April 2017 / Accepted: 6 July 2017 /Published online: 19 September 2017

(C) Springer-Verlag GmbH Germany 2017

\begin{abstract}
Aims/hypothesis The relative contribution of T helper (Th)1 and Th17 cells in graft rejection is inconclusive, on the basis of evidence provided by different $\mathrm{T}$ cell-related cytokine-deficient animal models and graft types.

Methods We used novel antigen-presenting-cell-specific $\mathrm{Il}$ $12 p 35$ (also known as $1112 a$ )-knockout (KO), IL-23p19knockdown (KD) and IL-27p28-KD strategies to investigate $\mathrm{T}$ cell differentiation in islet graft rejection.

Results In vitro dendritic cell-T cell coculture experiments revealed that dendritic cells from $\mathrm{Il}-12 \mathrm{p} 35-\mathrm{KO}$ and IL23p19-KD mice showed reduced ability to stimulate IFN- $\gamma$ and IL-17 production in T cells, respectively. To further explore the $\mathrm{T}$ cell responses in islet graft rejection, we transplanted islets into streptozotocin-induced diabetic NOD/ severe combined immunodeficiency (SCID) recipient mice with IL-12-, IL-23-, or IL-27-deficient backgrounds and then
\end{abstract}

Electronic supplementary material The online version of this article (https://doi.org/10.1007/s00125-017-4418-9) contains peer-reviewed but unedited supplementary material, which is available to authorised users.

Huey-Kang Sytwu

sytwu@ndmctsgh.edu.tw

1 Department and Graduate Institute of Microbiology and Immunology, National Defense Medical Center, 161, Section 6, MinChuan East Road, Neihu, Taipei 114, Taiwan

2 Graduate Institute of Life Sciences, National Defense Medical Center, Taipei, Taiwan

3 Department of Medicine, National Defense Medical Center, Taipei, Taiwan

4 Department of Biology and Anatomy, National Defense Medical Center, Taipei, Taiwan

5 Department of Internal Medicine, Tri-Service General Hospital, National Defense Medical Center, Taipei, Taiwan challenged them with NOD.BDC2.5 T cells. The survival of islet grafts was significantly prolonged in $I l-12 p 35-\mathrm{KO}$ and IL-23p19-KD recipients compared with the control recipients. $\mathrm{T}$ cell infiltrations and Th1 cell populations were also decreased in the grafts, correlating with prolonged graft survival. Conclusions/interpretation Our results suggest that IL-12 and IL-23 promote and/or maintain Th1 cell-mediated islet graft rejection. Thus, blockade of IL-12 and IL-23 might act as therapeutic strategies for reducing rejection responses.

Keywords Graft rejection · IL-12 · IL-23 · Islet transplantation $\cdot$ Th1 $\cdot$ Th17

$\begin{array}{ll}\text { Abbreviations } \\ \text { APC } & \text { Antigen-presenting cell } \\ \text { BMDC } & \text { Bone-marrow-derived dendritic cell } \\ \text { GIC } & \text { Graft-infiltrating cell } \\ \text { KD } & \text { Knockdown } \\ \text { KO } & \text { Knockout } \\ \text { PLN } & \text { Pancreatic lymph node } \\ \text { PRR } & \text { Pattern-recognition receptor } \\ \text { SCID } & \text { Severe combined immunodeficiency } \\ \text { Th } & \text { T helper } \\ \text { TLR } & \text { Toll-like receptor } \\ \text { Treg } & \text { Regulatory T } \\ \text { WT } & \text { Wild-type }\end{array}$

\section{Introduction}

Transplantation therapy is a promising method for curing many diseases, including cardiac vascular diseases, liver diseases and autoimmune diabetes. However, allorejection and recurrent autoimmunity are major obstacles in this therapy. In 
the early phase after organ or cell transplantation, the cytokine production profile is a critical factor in determining the fate of $\mathrm{T}$ helper (Th) cell responses and tipping the balance towards graft rejection or tolerance.

The differential activation of Th cells and the production of cytokines contribute to graft rejection or tolerance. Earlier studies have demonstrated that tolerising therapy prevents graft rejection by downregulating Th1 and Th1-related cytokine expression (IFN- $\gamma$ and IL-2) and increasing Th 2 cytokine expression (IL-4 and IL-10) in recipients [1-5], indicating that deviation from a Th1 to a Th2 cytokine profile in allograft recipients receiving tolerising therapy plays a crucial role in long-term engraftment [6]. Unexpectedly, further studies using Th1- and Th2-related cytokine knockout (KO) mice as recipients did not show prolonged survival or accelerated graft rejection, respectively $[7,8]$. This was probably because some cytokines have multiple functions that work at different stages or target different cells. For example, IL-2 signalling is essential for maintaining the homeostasis and competitive fitness of regulatory $\mathrm{T}$ (Treg) cells in vivo [9] and IFN- $\gamma$ is crucial for alloreactive Treg-cell-mediated suppression [10]. CD4 ${ }^{+} \mathrm{IL}-$ $17^{+}$(Th17) cells have been demonstrated to mediate allograft rejection in the absence of a Th1 cell response (T-bet [also known as $T b \times 21]-K O$ recipient) and neutralisation of IL-17 or IL-6 inhibits graft rejection $[11,12]$. However, these data cannot resolve the contribution of Th17 cells in a wild-type (WT) recipient carrying functional Th1 cells. Therefore, it became clear that the Th1/Th2 paradigm was insufficient to explain the mechanisms of graft rejection and the results obtained from different transplantation models (e.g. graft types, tolerising therapy strategies and various cytokine gene-KO recipients) are not conclusive; these are reviewed in Atalar et al, Liu et al and Askar et al [13-15].

Dendritic cells are central to the orchestration of host immunity against infectious agents and in self-tolerance. Dendritic cells express a panel of pattern-recognition molecules, which scavenge antigens and transmit danger signals to initiate an innate immune response. More importantly, dendritic cells act as a bridge that connects the innate and adaptive immune responses. In transplantation situations, the recipient's dendritic cells present the donor antigens to $\mathrm{T}$ cells and promote $\mathrm{T}$ cell differentiation via IL-12 family cytokines. IL12 and IL-23 are mainly proinflammatory and prostimulatory cytokines with key roles in the development of the Th1 and Th17 cell subsets, respectively [16]. IL-27 was thought initially to be a proinflammatory cytokine promoting early Th1 cell differentiation in the absence of IL-12 [17, 18] and further studies have demonstrated that IL-27 can suppress T cell hyperactivity and is involved in the induction of type 1 regulatory ( $\operatorname{Tr} 1)$ cells [19-22]. Together, these results highlight the importance of dendritic cells and IL-12 family cytokines in the initiation of Th cell activation and differentiation. Therefore, it is a reasonable approach to modulate the function of dendritic cells and monitor the subsequent effects on $\mathrm{T}$ cells, which could provide evidence to dissect the contribution of Th cell subsets in graft rejection and tolerance.

In this study, we used several genetically engineered mouse strains on a NOD background. NOD mice spontaneously develop autoimmune diabetes with immunopathological features that resemble human type 1 diabetes. We generated IL12-, IL-23- and IL-27-deficient mouse models to investigate the contribution of Th1 and Th17 cells in islet graft rejection. We hypothesised that different IL-12 family-deficient dendritic cells differentially drive Th cell differentiation, subsequently leading to islet graft destruction. This study provides new evidence to dissect the contributions of Th1 and Th17 cells in graft rejection, which might help the design of target therapies to reduce rejection responses.

\section{Methods}

Mice NOD, NOD/SCID (stock number 001303; mouse model of NOD and severe combined immunodeficiency [SCID]) and NOD.BDC2.5 (stock number 004460) mice were purchased initially from the Jackson Laboratory (Bar Harbor, ME, USA) and were subsequently bred and raised at the Animal Center of the National Defense Medical Center, which is accredited by the Association for Assessment and Accreditation of Laboratory Animal Care International (Taipei, Taiwan). All experiments were approved by the Institutional Animal Care and Use Committee Animal Center of the National Defense Medical Center. The IL-23p19-KD NOD mice, IL-27p28-KD NOD (ESM Methods) and Il-12p35 (also known as Il12a)-KO mice were previously generated in our laboratory [23]. These IL-12-family-deficient NOD mice were crossed with mice with a NOD/SCID background for transplantation experiments.

Dendritic cells and $\mathbf{T}$ cell coculture For $\mathrm{T}$ cell proliferation assays, $\mathrm{BDC} 2.5 \mathrm{CD} 4^{+} \mathrm{CD} 25^{-} \mathrm{T}$ cells $\left(10^{5}\right.$ cells $)$ were cocultured with dendritic cells $\left(5 \times 10^{3}\right.$ cells) from $I l-12 p 35$ KO, IL-23p19-KD or IL-27p28-KD mice in the presence of p31 peptide [24]. Cells were cultured in triplicate wells in flatbottomed 96 well plates. After $48 \mathrm{~h}$, the cultures were pulsed with $37 \mathrm{kBq}$ of $\left[{ }^{3} \mathrm{H}\right]$ methylthymidine (Amersham Pharmacia Biotech, Piscataway, NJ, USA); cells were harvested $16 \mathrm{~h}$ later onto UniFilter-96, GF/C filters (PerkinElmer, Boston, MA, USA), and the incorporated $\left[{ }^{3} \mathrm{H}\right]$ methylthymidine was detected using a TopCount counter (Packard, Meriden, CT, USA). For analysing $\mathrm{T}$ cell differentiation, $\mathrm{CD} 4^{+} \mathrm{CD} 25^{-} \mathrm{BDC} 2.5 \mathrm{~T}$ cells $\left(10^{6}\right.$ cells $)$ were cultured with splenic $\left(10^{6}\right.$ cells $)$ dendritic cells (WT, Il-12p35-KO, IL-23p19-KD and IL-27p28-KD) in the presence of $\mathrm{p} 31$ peptide $(0.2 \mu \mathrm{g} / \mathrm{ml})$ in 24 well plates for 3 days. T cells were harvested for flow cytometry and culture supernatant fractions were collected for ELISA. For isolation of T cell and dendritic cells, see ESM Methods. 
Islet transplantation and adoptive transfer Pancreatic islets were purified from 6 - to 8-week-old male NOD mice using the collagenase digestion method, as described previously [25]. Marginal islets were collected and 400 were implanted into the left renal capsule of mice with streptozotocin-induced diabetes: NOD/SCID and NOD/SCID with IL-12-familycytokine-deficient recipients (ESM Methods). The blood glucose concentration was monitored daily after islet transplantation and the recipients were rested for 1 week before adoptive transfer. For adoptive transfer, $\mathrm{CD} 4^{+} \mathrm{CD} 25^{-} \mathrm{T}$ cells or $\mathrm{CD}^{+} \mathrm{T}$ cells were isolated using BD IMag cell separation system (BD Pharmingen, San Jose, CA, USA). Normoglycaemic recipients received $2 \times 10^{5} \mathrm{CD} 4^{+} \mathrm{CD} 25^{-} \mathrm{T}$ cells from NOD.BDC2.5 mice ( $6 \sim 8$ weeks old) or a total of $2.5 \times 10^{6} \mathrm{CD}^{+} \mathrm{CD} 25^{-}$and $\mathrm{CD} 8^{+}$T cells (ratio 2:1) from NOD mice (6 8 weeks old), via intraperitoneal injection. Blood glucose concentration was monitored using a FreeStyle Optium Blood Glucose Monitoring System (Abbott, Abbott Park, IL, USA). Loss of graft function was defined as a blood glucose concentration $>16.65 \mathrm{mmol} / \mathrm{l}$ on two consecutive days.

Flow cytometry For analysis of the cell populations in reconstituted recipients, NOD/SCID, NOD/SCID Il$12 p 35-\mathrm{KO}, \mathrm{NOD} / \mathrm{SCID}$ IL-23p19-KD and NOD/SCID IL$27 \mathrm{p} 28-\mathrm{KD}$ recipients were perfused with $\mathrm{NaCl}$ solution (154 mmol/l) to remove blood cells and lymphocytes from the spleen and pancreatic lymph nodes (PLNs), and grafts were harvested for flow cytometry. For intracellular cytokine staining, cells were stimulated with phorbol 12-myristate 13acetate and ionomycin in the presence of monensin (all from Sigma-Aldrich, St Louis, MO, USA) for $4 \mathrm{~h}$, followed by intracellular cytokine staining. Cells were stained with antiCD4 (RM4-5), anti-CD8 (53-6.7), anti-CD25 (PC61.5), antiIFN- $\gamma$ (XMG1.2), anti-IL-17 (TC11-18H10.1) and antiforkhead box P3 ([FOXP3]; FJK-16s) antibodies (eBioscience, San Diego, CA, USA). Cells were analysed using a FACSCalibur flow cytometer and CellQuest software (BD Pharmingen). For characterization of the phenotype of the dendritic cells, see ESM Methods. All antibody staining procedures followed standard protocols provided by suppliers and each experiment included proper positive and negative controls.

Immunohistochemistry Graft-bearing kidneys were removed on day 11 after T cell transfer and embedded in optimal cutting temperature compound (OCT) (Leica Biosystems, Nussloch, Germany) for frozen sectioning (ESM Methods). The sections were stained with haematoxylin and eosin (Sigma-Aldrich). For immunohistochemistry, frozen tissue sections were stained with anti-insulin (1:50 dilution) (Abcam, Cambridge, UK) and anti-CD4 (1:200 dilution) (eBioscience, San Diego, CA, USA) primary antibodies and then with a horseradish peroxidase-conjugated secondary antibody; 3-amino-9-ethylcarbazole (Sigma-Aldrich) was added for enzymatic stain development. The images were captured via a Leica DM2500 microscope (Leica Biosystems).

ELISA Culture supernatant fractions from dendritic cell-T cell cocultures were analysed for levels of IFN- $\gamma$ and IL-17. All procedures followed the standard protocols provided by R\&D Systems (Minneapolis, MN, USA).

Statistics Prism version 6.00 software (GraphPad Prism, San Diego, CA, USA) was used for graph generation and statistical analysis and $p<0.05$ was defined as significant. Differences in cell population and cytokines production were determined by using $t$ tests and statistical significance levels were determined using the Holm-Sidak method. Survival statistics were analysed using the Kaplan-Meier method.

\section{Results}

Phenotypic characterisation of splenic lymphocytes and dendritic cells To evaluate the potential effects of the absence of these cytokines on dendritic cells and subsequent effects on other immune cells at steady state, we analysed the lymphocyte composition and characterised the phenotype of dendritic cells in WT, Il-12p35-KO, IL-23p19-KD, and IL-27p28-KD mice. The lymphocyte (ESM Fig. 1a) and Treg cell (ESM Fig. 1b) populations were not changed in these mice. To evaluate the effect of these cytokines on dendritic cells, we analysed the expressions of costimulatory/inhibitory molecules (CD40, CD80, CD86 and PD-L1), an adhesion molecule (CD54), lineage markers (CD103, DEC205 and 33D1) and MHC class II molecules on dendritic cells. The expression levels of these markers and the subsets of dendritic cells were not altered in the spleens of these mice (ESM Fig. 1c), suggesting that the lack of IL-12, IL-23 or IL-27 did not affect the function or development of dendritic cells in our models.

\section{Differential ability of dendritic cells to stimulate $\mathbf{T}$ cell pro-} liferation and differentiation Previous studies have demonstrated that IL-12 family cytokines are important for Th cell activation and differentiation, but how dendritic cells affect $\mathrm{CD}^{+} \mathrm{T}$ cells via these cytokines under rejection conditions is still unclear. We addressed this issue by establishing a dendritic cell-T cell coculture system in the presence of a specific antigen. To dissect the cellular mechanisms of $\mathrm{CD}^{+} \mathrm{T}$ cell proliferation, bone-marrow-derived dendritic cells (BMDCs) or splenic dendritic cells from WT, Il-12p35-KO, IL-23p19$\mathrm{KD}$ or IL-27p28-KD mice were cultured with $\mathrm{CD} 4^{+} \mathrm{CD} 25^{-} \mathrm{T}$ cells from NOD.BDC2.5 mice in the presence of $\mathrm{p} 31$ peptide at different concentrations. BMDCs and splenic dendritic cells from these cytokine-deficient mice induced antigen-specific $\mathrm{T}$ 
cell proliferation to similar levels as cells from WT mice (Fig. 1a-c and Fig. 1d, respectively), suggesting that deficiencies in these cytokines have a limited effect on T cell proliferation.

To investigate how cytokines of the IL-12 family affect antigen-dependent $\mathrm{CD} 4^{+} \mathrm{T}$ cell differentiation, we cocultured the splenic dendritic cells from WT, Il-12p35-KO, IL-23p19KD or IL-27p28-KD NOD/SCID mice with purified BDC2.5 $\mathrm{CD} 4^{+} \mathrm{CD} 25^{-} \mathrm{T}$ cells in the presence of the specific antigen, p31 peptide, to analyse differentiation in the Th cell populations. The dendritic cells from $\mathrm{Il}-12 \mathrm{p} 35-\mathrm{KO}$ mice displayed a reduced ability to promote IFN- $\gamma$ production by T cells (Fig. 2c). However, dendritic cells from IL-23p19-KD and IL27 p $28-K D$ mice showed similar capacity to induce $\mathrm{CD} 4^{+} \mathrm{IFN}-\gamma^{+}$(hereafter Th1) and $\mathrm{CD} 4^{+} \mathrm{IL}-17^{+}$(hereafter Th17) differentiation as the WT controls (Fig. 2a, b). Interestingly, ELISA analysis of supernatant fractions from the cocultures demonstrated that the IL-23p19-KD dendritic cells promoted IFN- $\gamma$ production and reduced IL-17 production (Fig. 2d). In contrast, Il-12p35-KO dendritic cells reduced IFN- $\gamma$ production but enhanced that of IL-17 (Fig. 2f). IL27p28-KD dendritic cells showed a reduced capacity only to promote IFN- $\gamma$ production (Fig. 2e). These results are consistent with previous reports that IL-12 and IL-27 promote Th1 cell differentiation and that IL-23 drives Th17 cell differentiation. Moreover, the IL-12/IFN- $\gamma$ pathway may counterregulate the IL-23/IL-17 pathways.

Survival of islet grafts after challenge with $T$ cells To investigate whether dendritic cells serve as primary targets to comprehensively investigate the contribution of Th cell subsets in graft rejection, we used IL-23p19-KD, IL-27p28-KD and Il$12 p 35-\mathrm{KO} \mathrm{NOD} / \mathrm{SCID}$ mice as recipients and then reconstituted these recipients with $\mathrm{T}$ cells. We used an islet transplantation model to investigate the contribution of Th cell subsets in graft rejection. We transplanted islets to Il-12p35KO, IL-23p19-KD or IL-27p28-KD diabetic NOD/SCID mice, and the normoglycaemic recipients then received $2 \times 10^{5} \mathrm{BDC} 2.5 \mathrm{CD} 4^{+} \mathrm{CD} 25^{-} \mathrm{T}$ cells. The survival of islet grafts was prolonged in the $I l-12 p 35-\mathrm{KO}$ (mean graft survival 49 days) and IL-23p19-KD recipients (mean graft survival 26 days), whereas graft survival was not altered in IL27p28-KD mice after diabetogenic T cell transfer (mean graft survival 13 days) compared with the control recipients (mean graft survival 12 days) (Fig. 3). However, islet grafts were rejected with similar kinetics in the IL-23p19-KD $\times \mathrm{Il}$ $12 p 35-\mathrm{KO}$ recipients compared with the Il-12p35-KO recipients, indicating that IL-12 plays a dominant role in islet graft rejection. We also performed histology on islet grafts at day 11 to evaluate graft function and T cell infiltration (Fig. 4). The grafts from IL-23p19-KD and Il-12p35-KO mice showed more intact islet morphology (Fig. 4) and less CD4 T cell infiltration (Fig. 4), consistent with high levels of insulin (Fig. 4). The grafts recovered from $I l-12 p 35-\mathrm{KO}$ and IL23p19-KD recipients displayed less severe $\mathrm{T}$ cell infiltration than those from the WT recipients, and this was positively associated with graft survival. However, grafts from IL$27 \mathrm{p} 28-\mathrm{KD}$ recipients displayed a level of infiltration similar to that in the WT group.

Th cell differentiation and dendritic cell characterisation in reconstituted recipients To investigate the contribution of T cell populations to graft rejection, we transferred polyclonal $\mathrm{CD} 4^{+} \mathrm{CD} 25^{-}$cells together with $\mathrm{CD} 8^{+} \mathrm{T}$ cells into recipients and analysed the activation status of $\mathrm{T}$ cells on days 14-16 after cell transfer. We isolated cells from graft (graft-infiltrating cells [GICs]), spleens and PLNs (the primary homing
Fig. 1 The ability of dendritic cells from IL-12-family-deficient mice to stimulate $\mathrm{T}$ cell proliferation. $\mathrm{CD} 4^{+} \mathrm{CD} 25^{-} \mathrm{T}$ cells isolated from NOD.BDC2.5 mice were cultured with BMDCs from NOD mice $(\mathbf{a}-\mathbf{c})$ or splenic dendritic cells from NOD/SCID mice (d) in the presence of $\mathrm{p} 31$ peptide at the concentrations indicated. Data are presented as the mean \pm standard error of the mean. Representative results are shown from at least three independent experiments. Black, WT; white, IL23p19-KD; light grey, IL-27p28$\mathrm{KD}$; dark grey, $\mathrm{Il}-12 \mathrm{p} 35-\mathrm{KO}$
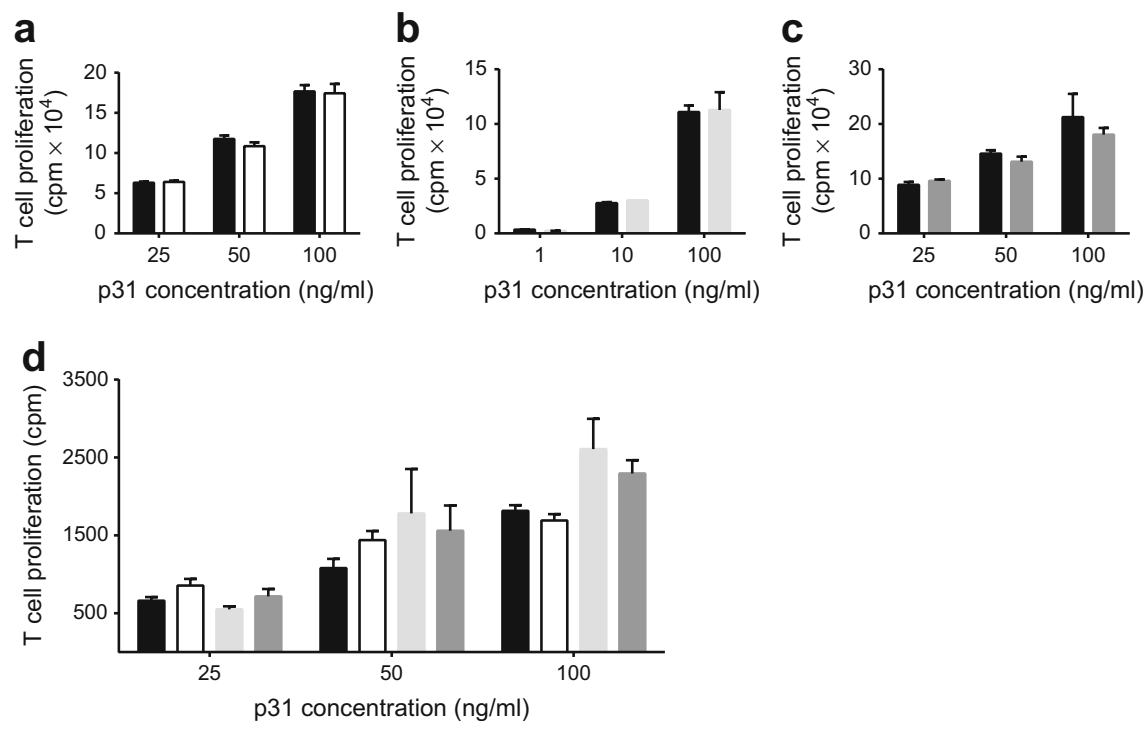

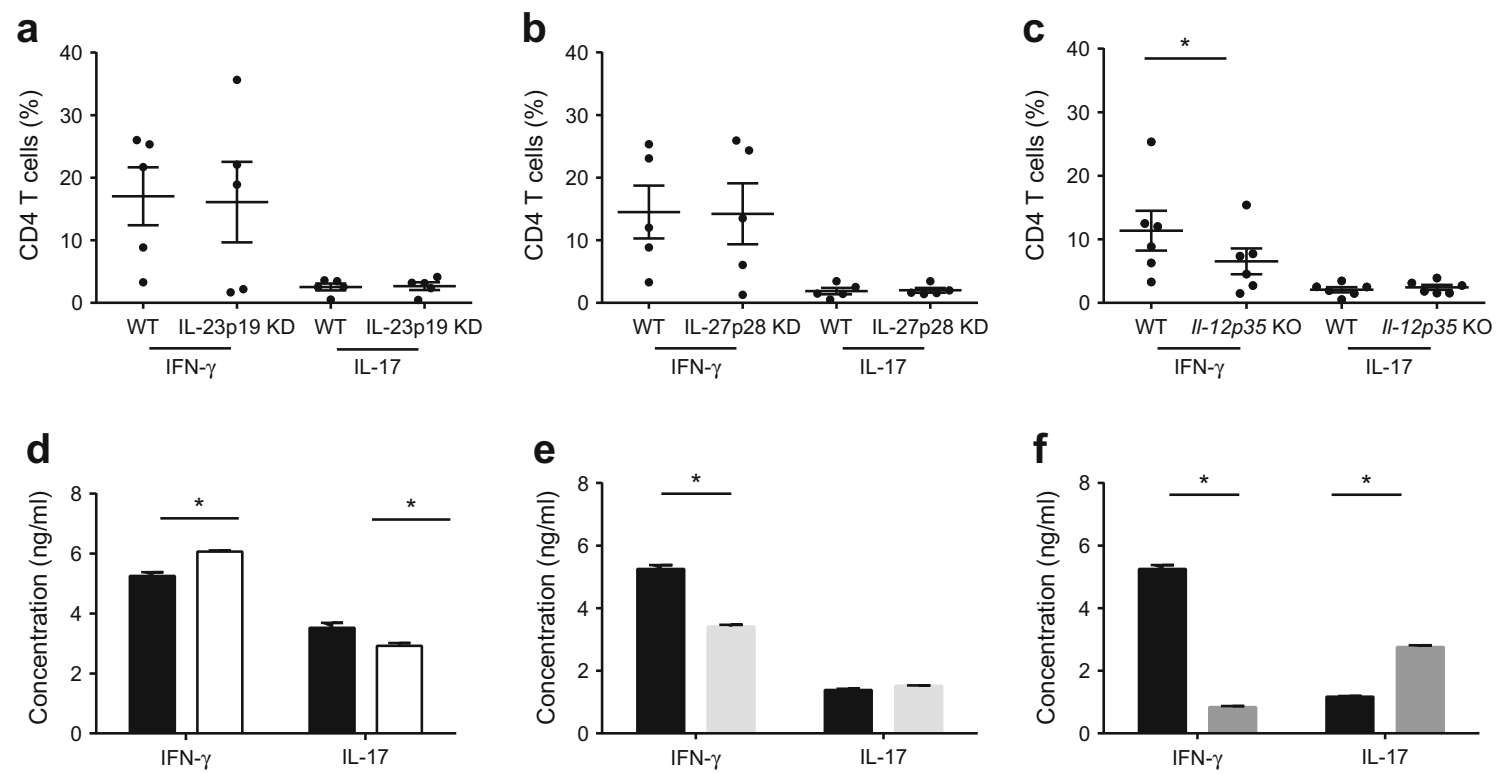

Fig. 2 The effects of dendritic cells deficient in cytokines of the IL-12 family in driving $\mathrm{T}$ cell differentiation and cytokine production. $\mathrm{CD} 4^{+} \mathrm{CD} 25^{-} \mathrm{T}$ cells isolated from NOD.BDC2.5 mice were cocultured with splenic dendritic cells from various IL-12-family-deficient NOD/ SCID or WT control mice in the presence of $\mathrm{p} 31$ peptide $(0.2 \mu \mathrm{g} / \mathrm{ml})$ for 3 days. T cells were harvested for immunostaining for intracellular

cytokines and flow cytometry $(\mathbf{a}-\mathbf{c})$. Culture supernatant fractions were analysed by ELISA (d-f). (a, d) WT vs IL-23p19-KD. (b, e) WT vs IL$27 \mathrm{p} 28-\mathrm{KD}$. (c, f) WT vs $I 112 p 35 \mathrm{KO} .{ }^{*} p<0.05$ compared with the WT group. Representative results are shown from at least seven independent experiments; $(\mathbf{a}-\mathbf{c})$ paired $t$ tests; $(\mathbf{d}-\mathbf{f})$ unpaired $t$ tests. Black, WT; white, IL-23p19-KD; light grey, IL-27p28-KD; dark grey, Il-12p35- KO

lymph nodes of diabetogenic lymphocytes, used as a control) and analysed the cell populations and cytokine production. The total number of GICs from Il-12p35-KO recipients was significantly lower than from the WT recipients; however, cell counts from the other groups did not show significant differences from the WT control group (Table 1). We analysed the populations of effector cells and Treg cells in these recipients. The IFN- $\gamma^{+}, \mathrm{IL}-17^{+}$and IFN ${ }^{+} \mathrm{IL}-17^{+} \mathrm{CD} 4 \mathrm{~T}$ cell populations were similar in the spleens and PLNs of the recipients (Fig. $5 \mathrm{a}-\mathrm{f}$ ), even though the IFN $-\gamma^{+} \mathrm{CD} 4^{+}$population was decreased in the spleens of IL-23p19-KD recipients (Fig. 5a). Importantly, the IFN $-\gamma^{+} \mathrm{CD} 4^{+}$population was decreased

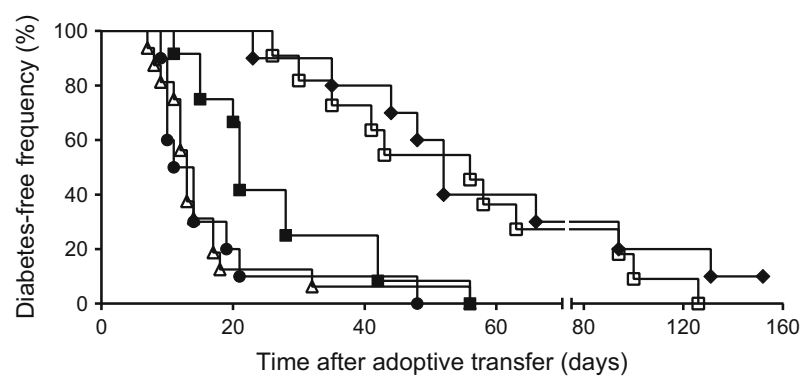

Fig. 3 Islet graft survival in NOD/SCID recipients after challenge with BDC2.5 CD4 ${ }^{+} \mathrm{CD} 25^{-}$T cells. The Kaplan-Meier method was used for analysis. WT vs $I l-12 p 35 \mathrm{KO}, p<0.0001$; WT vs IL23p19-KD, $p=0.0024$; WT vs IL-27p28-KD, $p=0.9923$; WT vs IL-23p19-KD $\times$ Il-12p35-KO, $p=0.0005$; and Il-12p35-KO vs IL-23p19-KD, $p=0.0013$. White triangles, WT; white squares, $I l-12 p 35-\mathrm{KO}$; black squares, IL23p19-KD; black circles, IL-27p28-KD; black diamonds, IL-23p19$\mathrm{KD} \times \mathrm{Il}-12 \mathrm{p} 35-\mathrm{KO}$ in the grafts of $I l-12 p 35-\mathrm{KO}$ and IL-23p19-KD recipients, which showed prolonged graft survival (Fig. $5 \mathrm{~g}-\mathrm{i}$ ). In addition, IFN- $\gamma^{+}$cells were more frequent than IL- $17^{+}$cells in this model, suggesting that Th1 cells play a crucial role in islet graft rejection and that the lack of IL-12 mitigates detrimental Th1 cell-mediated rejection. These results demonstrate that Th1 cells play dominant roles in islet graft destruction and that IL-12 and IL-23 are necessary for the generation or maintenance of graft-specific Th1 cell responses.

As Treg cells play crucial roles in transplant tolerance, we further checked the $\mathrm{CD}^{+}{ }^{+} \mathrm{FOXP}^{+}$regulatory $\mathrm{T}$ cell population in recipients. There were no significant changes in the Treg cell population in the spleens, PLNs or grafts (Fig. 5j), suggesting that our IL-12-family-cytokine-deficient mouse model was suitable for the study of effector $\mathrm{T}$ cell differentiation during graft rejection and that the results might not have been affected by Treg cells.

Finally, we checked the populations and status of the $\mathrm{CD} 11 \mathrm{c}^{+}$dendritic cells in recipients (ESM Fig. 2). The expression levels of CD40, CD80, CD86, CD54, PD-L1 and MHC class II molecules were not altered in these IL-12-familycytokine-deficient recipients. The dendritic cell subsets were also similar among these recipients. These results indicate that lack of IL-12, IL-23 and IL-27 might not alter the development or surface molecule expression of dendritic cells in the recipients; however, IL-12 and IL-23 secreted by dendritic cells promote $\mathrm{T}$ cell differentiation and function, leading to graft rejection. 
Fig. 4 Histology and immunohistochemistry of islet grafts under the capsule of the kidney. Islet graft sections were stained with haematoxylin and eosin (a), or immunohistochemically stained using anti-insulin (b) or anti-CD4 (c) antibodies to evaluate the architecture, function and $\mathrm{T}$ cell infiltration of the islet graft. Rosered to brownish-red colours in (b) and (c) indicate insulin and CD4 expression, respectively. Scale bar, $200 \mu \mathrm{m}$. HE, haematoxylin and eosin

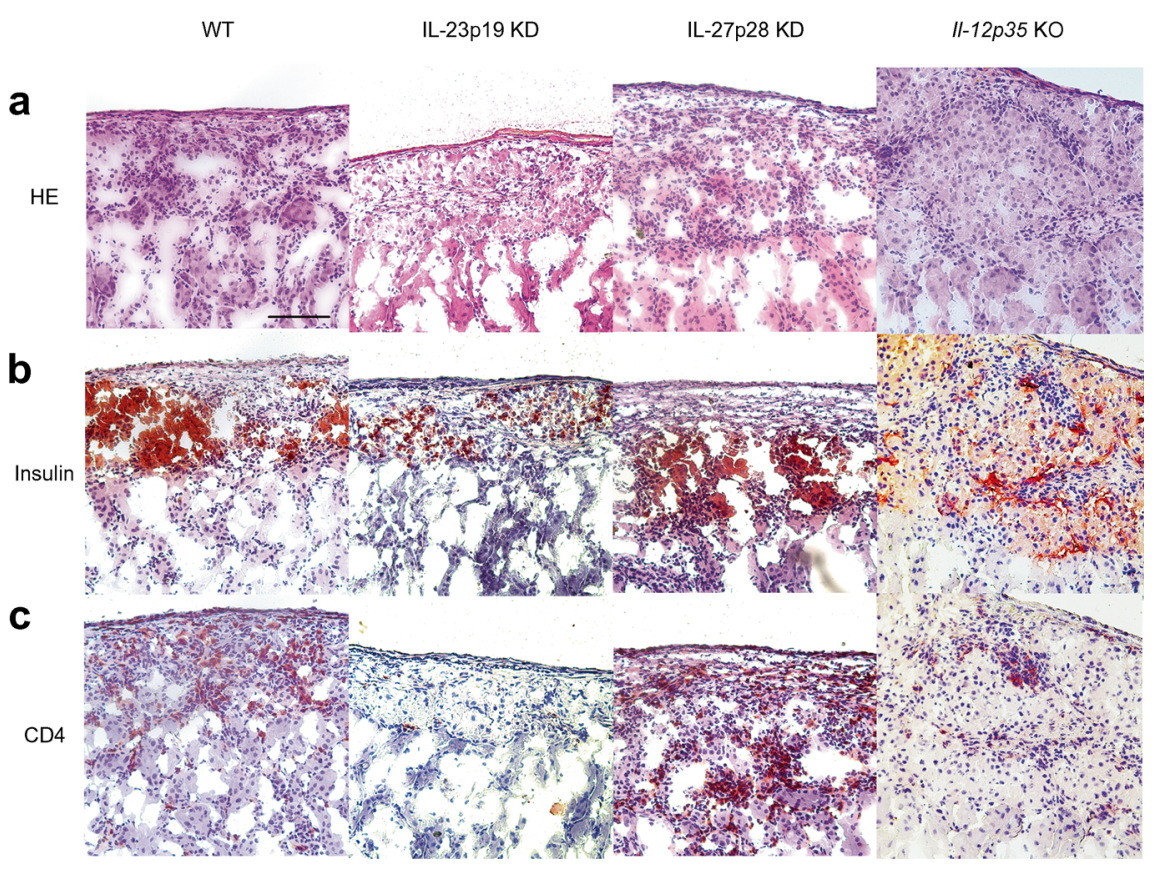

\section{Discussion}

The contributions of Th cell subsets to graft rejection have been investigated intensively in various graft tissues using different Th cell-related effector cytokines or models with knockout of genes for lineage-specific transcription factors (e.g. IL-2, IL-10, IFN- $\gamma$, IL-17, T-box 21 [T-bet] and RARrelated orphan receptor gamma $\mathrm{t}[\mathrm{ROR} \gamma \mathrm{t}])[7,8,11,26]$. Although the results obtained using these models highlight the contribution of specific $\mathrm{T}$ cell populations in graft rejection, the full orchestra of $\mathrm{T}$ cell immune responses might be missed because of the multiple functions of cytokines and their compensatory effects. In this study, we used models deficient in cytokines of the IL-12 family to investigate the influence of Th cell differentiation and inflammatory processes, providing different angles from which to understand rejection responses. We found that the proinflammatory cytokines IL12 and IL-23 were crucial in transplant rejection, promoting

Table 1 Cell numbers in the spleens, PLNs and GICs of IL-12-familycytokine-deficient recipients

\begin{tabular}{lrrl}
\hline Mouse & \multicolumn{2}{l}{ Absolute cell numbers $\left(\times 10^{4}\right)$} \\
\cline { 2 - 4 } & \multicolumn{1}{l}{ Spleen } & \multicolumn{1}{l}{ PLN } & \multicolumn{1}{l}{ GIC } \\
\hline WT & $1360 \pm 295$ & $19 \pm 4$ & $288 \pm 27$ \\
IL-23p19-KD & $1673 \pm 360$ & $34 \pm 8$ & $221 \pm 25$ \\
IL-27p28-KD & $853 \pm 163$ & $7 \pm 3$ & $280 \pm 49$ \\
Il-12p35-KO & $1763 \pm 278$ & $18 \pm 8$ & $175 \pm 38^{*}$ \\
\hline
\end{tabular}

${ }^{*} p<0.05$ compared with WT group, unpaired $t$ test effector Th1 and-to a lesser extent-Th17 responses. Previous studies have demonstrated that the IL-12-Th1 axis cross-regulates the IL-23-Th17 axis [27, 28], as confirmed in our in vitro coculture experiments. However, our in vivo results revealed that both IL-12 and IL-23 were critical in promoting or maintaining Th1 cell function in the grafts, suggesting that the actions of these cytokines are context dependent.

The reduction in Th1 cells in IL-23p19-KD recipients was unexpected. However, this might be explained by a finding that IL-23 mainly acts on effector/memory cells [29], so a reduced IL-23 level in vivo might diminish the effector cell population. Although the Th17 cell population was only slightly decreased (Fig. 5a-c), previous reports have demonstrated that IL-17 enhances IFN- $\gamma+$ IL-1 $\beta$-induced islet apoptosis and chemokine expression [30]. Thus, these mechanisms may account for the prolonged graft survival in the IL-23-KD recipients. Besides, it is unexpected that islet graft survival was not further prolonged in IL-23p19-KD $\times$ Il$12 p 35-\mathrm{KO}$ recipients, suggesting that the IL-12-Th1 axis plays a crucial role in islet graft rejection. As IL-27 plays both proinflammatory and anti-inflammatory roles, its net effect was no influence on either graft rejection or tolerance in our islet transplantation model.

Previous studies have demonstrated that dendritic cells also express receptors for cytokines of the IL-12 family and that the function of dendritic cells could be modulated by these cytokines with lipopolysaccharide stimulation or experimental autoimmune encephalomyelitis [31-35]. By analysing the phenotype of splenic dendritic cells from NOD and NOD/ SCID recipients with background cytokine deficiencies, we found that there were no differences in dendritic cell 

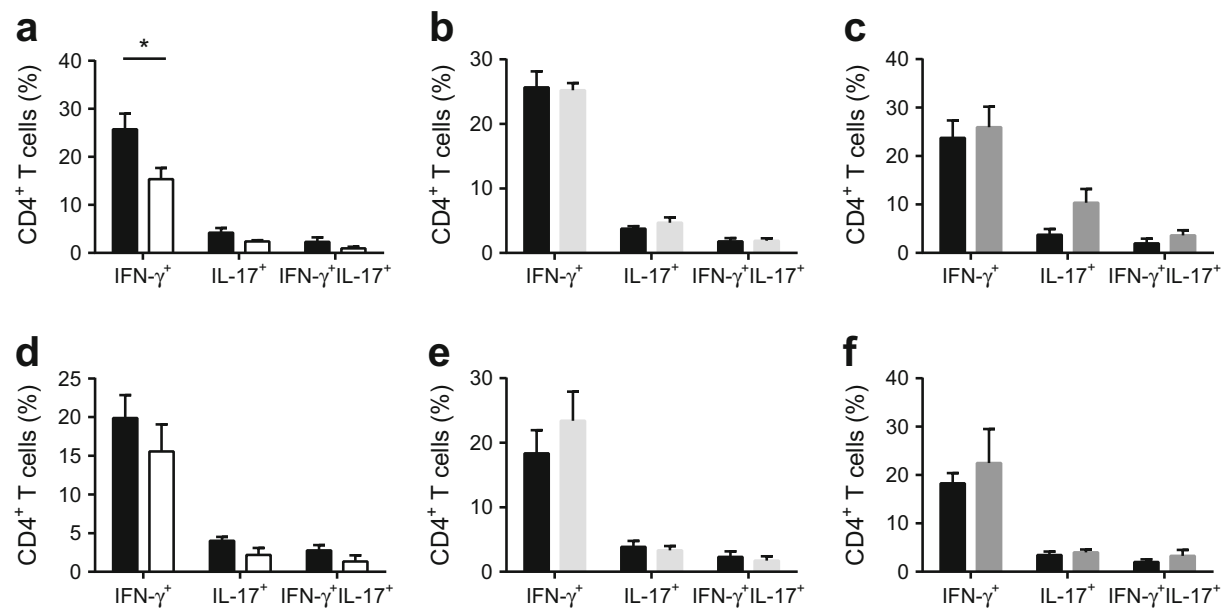
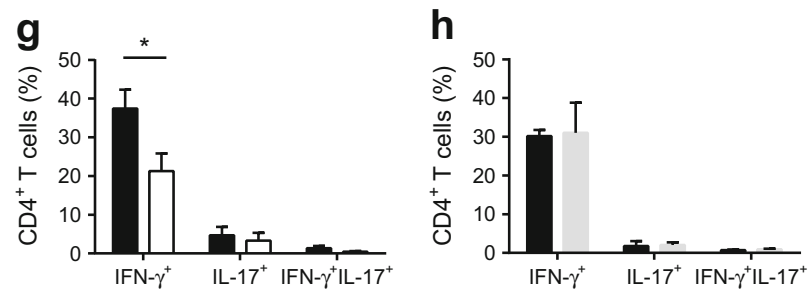

Fig. 5 Flow cytometry analysis of the T cell populations in recipients after challenge with $T$ cells. Lymphocytes from $(\mathbf{a}-\mathbf{c})$ the spleen, $(\mathbf{d}-\mathbf{f})$ PLNs and (g-i) GICs of NOD/SCID recipients were isolated and analysed using flow cytometry. (j) $\mathrm{CD} 4^{+} \mathrm{CD} 25^{+} \mathrm{FOXP} 3^{+} \mathrm{T}$ cells in the

phenotypes among the Il-12p35-KO, IL-23p19-KD and IL$27 \mathrm{p} 28-\mathrm{KD}$ mice. Our results suggest that deficiencies in cytokines of the IL-12 family mainly affect $\mathrm{T}$ cell differentiation rather than the dendritic cells themselves.

Dendritic cells express a panel of pattern-recognition receptors (PRRs) that sense danger signals to induce inflammation. The danger signals evoked though PRRs induce various cytokines of the IL-12 family that influence the development of Th1 and Th17 cell subsets. It has been hypothesised that allografts of tissues (e.g. skin, intestines and lungs) routinely exposed to pathogens and commensal organisms might display reduced susceptibility to transplant acceptance [36]. This idea has been demonstrated in mouse skin and cardiac transplantation models showing that the engagement of Toll-like receptors (TLRs) abolishes the induction of tolerance [37, 38]. In the early phase after transplantation, the activation of TLRs by endogenous ligands released during ischaemia-reperfusion injury is also thought to play an important role in dendritic cell activation, leading to the production of cytokines responsible for the polarisation of $\mathrm{T}$ cell responses [39]. Together, these results highlight the importance of dendritic cells in the initiation of Th cell activation and differentiation. Consequently, it is reasonable to modulate the function of dendritic cells experimentally and monitor the subsequent effects on T cells, which could provide the evidence needed to clarify the contribution of Th cell subsets in graft rejection and tolerance.
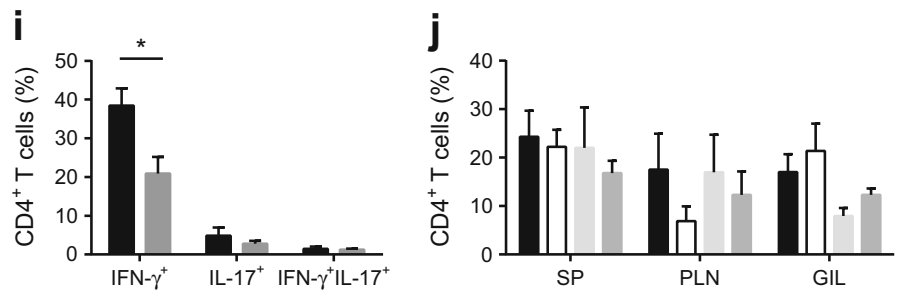

spleen, PLNs and GICs. Combined results are from 3-5 mice for each group (mean \pm standard error of the mean). ${ }^{*} p<0.05$ compared with the WT control group by unpaired $t$ test. Black, WT; white, IL-23p19-KD; light grey, IL-27p28-KD; dark grey, Il-12p35-KO

Reports that pretreatment of grafts with ultraviolet or gamma irradiation reduces graft immunogenicity and prolongs graft survival in rat allotransplantation models emphasise the importance of donor antigen-presenting cells (APCs) in graft rejection $[40,41]$. In many mouse strains, including NOD mice, dendritic cells are present in the islet and constitutively present beta cell-derived peptides [42]. In this study, we investigated the effects of the recipient's APCs on islet graft rejection (indirect presentation). However, our models cannot rule out a role for donor islet APCs in the mediation of direct antigen presentation and $T$ cell activation, which may promote graft rejection. Islets from donors deficient in IL-12 family cytokines may have had longer graft survival times than in the current setting. Furthermore, we have carried out allogeneic skin transplantation experiments using NOD mice deficient in IL-12 family cytokines as recipients. We found that skin grafts to these deficient recipients were rejected with similar kinetics to WT recipient rejection (F-C Chou and HY Chen, unpublished data). These data suggested that resident APCs within the donor tissue also play a crucial role in the initial graft rejection and the contribution of donor APCs is context dependent.

In conclusion, the results obtained in this study could help inform preventive and/or therapeutic strategies for transplantation that involve manipulating the expression of immunomodulatory cytokines in dendritic cells, and the induction of 
tolerance in genetically modified dendritic cells in models of autoimmune diabetes. Moreover, understanding the mechanisms that control the cytokines produced by dendritic cells and subsequently promote Th cell differentiation will provide more insight to transplantation immunology. We could also design therapies that block specific subunits of selective IL12 family cytokines to interrupt early Th cell differentiation after transplantation. Furthermore, these animal models could be applied further to study other types of transplanted tissues (e.g. kidney and heart) and thereby help elucidate the role of Th cell subsets in the rejection response.

Acknowledgements Some of the data were presented as an abstract at the 63rd Annual Meeting of the Japanese Association for Laboratory Animal Science, 18-20 May 2016, Kawasaki, Japan, and the 16th International Congress of Immunology, 21-26 August 2016, Melbourne, Australia.

Data availability The datasets generated during and/or analysed during the current study are available from the corresponding author on reasonable request.

Funding This work was supported by the Ministry of Science and Technology (MOST), Taiwan, Republic of China. (MOST 103-2320-B016-017-MY3 and MOST 104-2320-B-016-014-MY3 to HKS; NSC 102-2321-B-016-005-MY3 to FCC), Tri-Service General Hospital Foundation (TSGH-C106-004-006-008-S02 to HKS), and Tri-Service General Hospital (VTA106-T-1-1 to HKS).

Duality of interest The authors confirm that there is no duality of interest associated with this manuscript.

Contribution statement FCC, HYC, HHC, GJL, SHL and HKS contributed to conception and design, acquisition of data or analysis and interpretation of data. All authors drafted the article and/or revised it critically for important intellectual content. All authors approved the final version to be published. FCC and HKS are responsible for the integrity of the work as a whole.

\section{References}

1. Takeuchi T, Lowry RP, Konieczny B (1992) Heart allografts in murine systems. The differential activation of Th2-like effector cells in peripheral tolerance. Transplantation 53:1281-1294

2. Sayegh MH, Akalin E, Hancock WW et al (1995) CD28-B7 blockade after alloantigenic challenge in vivo inhibits Th1 cytokines but spares Th2. J Exp Med 181:1869-1874

3. Nickerson P, Pacheco-Silva A, O'Connell PJ, Steurer W, Kelley VR, Strom TB (1993) Analysis of cytokine transcripts in pancreatic islet cell allografts during rejection and tolerance induction. Transplant Proc 25:984-985

4. Mottram PL, Han WR, Purcell LJ, McKenzie IF, Hancock WW (1995) Increased expression of IL-4 and IL-10 and decreased expression of IL-2 and interferon-gamma in long-surviving mouse heart allografts after brief CD4-monoclonal antibody therapy. Transplantation 59:559-565
5. Hancock WW, Sayegh MH, Kwok CA, Weiner HL, Carpenter CB (1993) Oral, but not intravenous, alloantigen prevents accelerated allograft rejection by selective intragraft Th2 cell activation. Transplantation 55:1112-1118

6. Strom TB, Roy-Chaudhury P, Manfro R et al (1996) The Th1/Th2 paradigm and the allograft response. Curr Opin Immunol 8:688693

7. Steiger J, Nickerson PW, Steurer W, Moscovitch-Lopatin M, Strom TB (1995) IL-2 knockout recipient mice reject islet cell allografts. J Immunol 155:489-498

8. Raisanen-Sokolowski A, Mottram PL, Glysing-Jensen T, Satoskar A, Russell ME (1997) Heart transplants in interferon-gamma, interleukin 4, and interleukin 10 knockout mice. Recipient environment alters graft rejection. J Clin Invest 100:2449-2456

9. Fontenot JD, Rasmussen JP, Gavin MA, Rudensky AY (2005) A function for interleukin 2 in Foxp3-expressing regulatory $\mathrm{T}$ cells. Nat Immunol 6:1142-1151

10. Sawitzki B, Kingsley CI, Oliveira V, Karim M, Herber M, Wood KJ (2005) IFN-gamma production by alloantigen-reactive regulatory $\mathrm{T}$ cells is important for their regulatory function in vivo. J Exp Med 201:1925-1935

11. Yuan X, Paez-Cortez J, Schmitt-Knosalla I et al (2008) A novel role of CD4 Th17 cells in mediating cardiac allograft rejection and vasculopathy. J Exp Med 205:3133-3144

12. Burrell BE, Csencsits K, Lu G, Grabauskiene S, Bishop DK (2008) CD8+ Th17 mediate costimulation blockade-resistant allograft rejection in T-bet-deficient mice. J Immunol 181:3906-3914

13. Atalar K, Afzali B, Lord G, Lombardi G (2009) Relative roles of Th1 and Th17 effector cells in allograft rejection. Curr Opin Organ Transplant 14:23-29

14. Liu Z, Fan H, Jiang S (2013) CD4(+) T cell subsets in transplantation. Immunol Rev 252:183-191

15. Askar M (2014) T helper subsets \& regulatory T cells: rethinking the paradigm in the clinical context of solid organ transplantation. Int J Immunogenet 41:185-194

16. Hunter CA (2005) New IL-12-family members: IL-23 and IL-27, cytokines with divergent functions. Nat Rev Immunol 5:521-531

17. Owaki T, Asakawa M, Morishima N et al (2005) A role for IL-27 in early regulation of Th1 differentiation. J Immunol 175:2191-2200

18. Zhu J, Jankovic D, Oler AJ et al (2012) The transcription factor Tbet is induced by multiple pathways and prevents an endogenous Th2 cell program during Th1 cell responses. Immunity 37:660-673

19. Yoshida H, Nakaya M, Miyazaki Y (2009) Interleukin 27: a doubleedged sword for offense and defense. J Leukoc Biol 86:1295-1303

20. Awasthi A, Carrier Y, Peron JP et al (2007) A dominant function for interleukin 27 in generating interleukin 10-producing anti-inflammatory T cells. Nat Immunol 8:1380-1389

21. Batten M, Li J, Yi S et al (2006) Interleukin 27 limits autoimmune encephalomyelitis by suppressing the development of interleukin 17-producing T cells. Nat Immunol 7:929-936

22. Villarino A, Hibbert L, Lieberman L et al (2003) The IL-27R (WSX-1) is required to suppress T cell hyperactivity during infection. Immunity 19:645-655

23. Hung JT, Liao JH, Lin YC et al (2005) Immunopathogenic role of TH1 cells in autoimmune diabetes: evidence from a T1 and T2 doubly transgenic non-obese diabetic mouse model. J Autoimmun 25:181-192

24. Judkowski V, Pinilla C, Schroder K, Tucker L, Sarvetnick N, Wilson DB (2001) Identification of MHC class II-restricted peptide ligands, including a glutamic acid decarboxylase 65 sequence, that stimulate diabetogenic $\mathrm{T}$ cells from transgenic BDC2.5 nonobese diabetic mice. J Immunol 166:908-917

25. Chou FC, Kuo CC, Wang YL et al (2013) Overexpression of galectin-9 in islets prolongs grafts survival via downregulation of Th1 responses. Cell Transplant 22:2135-2145 
26. Sabet-Baktach M, Eggenhofer E, Rovira J et al (2013) Double deficiency for RORgammat and T-bet drives Th2-mediated allograft rejection in mice. J Immunol 191:4440-4446

27. Cruz A, Khader SA, Torrado E et al (2006) Cutting edge: IFNgamma regulates the induction and expansion of IL-17-producing CD4 T cells during mycobacterial infection. J Immunol 177:14161420

28. Harrington LE, Hatton RD, Mangan PR et al (2005) Interleukin 17producing CD4+ effector T cells develop via a lineage distinct from the $T$ helper type 1 and 2 lineages. Nat Immunol 6:1123-1132

29. Oppmann B, Lesley R, Blom B et al (2000) Novel p19 protein engages IL-12p40 to form a cytokine, IL-23, with biological activities similar as well as distinct from IL-12. Immunity 13:715-725

30. Grieco FA, Moore F, Vigneron F et al (2014) IL-17A increases the expression of proinflammatory chemokines in human pancreatic islets. Diabetologia 57:502-511

31. Zhang GX, Yu S, Gran B et al (2003) Role of IL-12 receptor beta 1 in regulation of $\mathrm{T}$ cell response by APC in experimental autoimmune encephalomyelitis. J Immunol 171:4485-4492

32. Zhang GX, Gran B, Yu S et al (2003) Induction of experimental autoimmune encephalomyelitis in IL-12 receptor-beta 2deficient mice: IL-12 responsiveness is not required in the pathogenesis of inflammatory demyelination in the central nervous system. J Immunol 170:2153-2160

33. Awasthi A, Riol-Blanco L, Jager A et al (2009) Cutting edge: IL-23 receptor gfp reporter mice reveal distinct populations of IL-17producing cells. J Immunol 182:5904-5908
34. Mascanfroni ID, Yeste A, Vieira SM et al (2013) IL-27 acts on dendritic cells to suppress the $\mathrm{T}$ cell response and autoimmunity by inducing expression of the immunoregulatory molecule CD39. Nat Immunol 14:1054-1063

35. Wang S, Miyazaki Y, Shinozaki Y, Yoshida H (2007) Augmentation of antigen-presenting and Th1-promoting functions of dendritic cells by WSX-1(IL-27R) deficiency. J Immunol 179:6421-6428

36. Alegre ML, Chong A (2009) Toll-like receptors (TLRs) in transplantation. Front Biosci 1:36-43

37. Chen L, Wang T, Zhou P et al (2006) TLR engagement prevents transplantation tolerance. Am J Transplant 6:2282-2291

38. Chen L, Ahmed E, Wang T et al (2009) TLR signals promote IL-6/ IL-17-dependent transplant rejection. J Immunol 182:6217-6225

39. Leemans JC, Stokman G, Claessen N et al (2005) Renal-associated TLR2 mediates ischemia/reperfusion injury in the kidney. J Clin Invest 115:2894-2903

40. Lau H, Reemtsma K, Hardy MA (1984) Prolongation of rat islet allograft survival by direct ultraviolet irradiation of the graft. Science 223:607-609

41. James RF, Lake SP, Chamberlain J et al (1989) Gamma irradiation of isolated rat islets pretransplantation produces indefinite allograft survival in cyclosporine-treated recipients. Transplantation 47:929 933

42. Calderon B, Suri A, Miller MJ, Unanue ER (2008) Dendritic cells in islets of Langerhans constitutively present beta cell-derived peptides bound to their class II MHC molecules. Proc Natl Acad Sci U S A 105:6121-6126 